
\title{
R Research Sourere \\ Unexpected Similarity Between HIV囚1 Reverse Transcriptase and Tumor Necrosis Factor Binding Sites Revealed by Computer Vision.
}

\section{Merveille Eguida}

University of Strasbourg: Universite de Strasbourg https://orcid.org/0000-0002-0976-0239

Didier Rognan ( $\square$ rognan@unistra.fr)

University of Strasbourg: Universite de Strasbourg https://orcid.org/0000-0002-0577-641X

\section{Research Article}

Keywords: binding sites, similarity, point cloud registration

Posted Date: August 30th, 2021

DOI: https://doi.org/10.21203/rs.3.rs-820779/v1

License: (c) (1) This work is licensed under a Creative Commons Attribution 4.0 International License.

Read Full License

Version of Record: A version of this preprint was published at Journal of Cheminformatics on November 23rd, 2021. See the published version at https://doi.org/10.1186/s13321-021-00567-3. 
1 Unexpected similarity between HIV-1 reverse transcriptase and tumor necrosis

2 factor binding sites revealed by computer vision.

3

4 Merveille Eguida ${ }^{1}$ and Didier Rognan ${ }^{1, *}$

5

$6{ }^{1}$ Laboratoire d'Innovation Thérapeutique, UMR 7200 CNRS-Université de Strasbourg, 67400 IIIkirch,

7 France.

8

9

$10 *$ Corresponding author

11 E-mail: rognan@unistra.fr

12

13 
15 Rationalizing the identification of hidden similarities across the repertoire of druggable protein cavities remains a major hurdle to a true proteome-wide structure-based discovery of novel drug candidates. We recently described a new computational approach (ProCare), inspired by numerical image processing, to identify local similarities in fragment-based subpockets. During the validation of the method, we unexpectedly identified a possible similarity in the binding pockets of two unrelated targets, human tumor necrosis factor alpha (TNF- $\alpha$ ) and HIV-1 reverse transcriptase (HIV-1 RT). Microscale thermophoresis experiments confirmed the ProCare prediction as two of the three tested and FDA-approved HIV-1 RT inhibitors indeed bind to soluble human TNF- $\alpha$ trimer. Interestingly, the herein disclosed similarity could be revealed neither by state-of-the-art binding sites comparison methods nor by ligand-based pairwise

24 similarity searches, suggesting that the point cloud registration approach implemented in ProCare, is uniquely suited to identify local and unobvious similarities among totally unrelated targets. 


\section{INTRODUCTION}

Among the many possible approaches to structure-based drug design $[1,2]$, inferring novel ligand properties from the large-scale comparison of their possible binding pockets gains popularity as the repertoire of protein cavities of known three-dimensional (3D) structures (pocketome) is constantly increasing, thereby offering unique opportunities to design ligands while simultaneously considering multiple targets [3]. The term 'pocketome' was first coined in 2004 by An et al. [4] to describe the universe of cavities located at the surface of macromolecules and capable of binding low molecular-weight ligands. A systematic survey of currently available three-dimensional structures [5], suggests that its size is estimated to ca. 250,000 pockets [6] out of which $10-15 \%$ are accommodating true drug-like compounds $[7,8]$. Pocket locations can be inferred from the position of already-bound molecules or predicted on the fly, by one of the many available cavity detection methods $[3,9]$. The pockeome space can then be searched by numerous computational tools [10] for similarity to any query cavity to predict evolutionary relationships and protein-ligand interactions [3]. The later application is notably of paramount importance to the drug discovery field as it may reveal hidden relationships for guiding the design of safer drug candidates with a precise control of selectivity [3] with respect to either on-targets (polypharmacology approach) [11] or off-targets (side effects mitigation) [12], in a time and cost-effective manner [13].

Currently available methods are generally able to detect global similarities between two druggable pockets from different proteins, and therefore permit to transfer drug-like compounds from one target space to another [3]. Identifying more subtle local similarities at the level of fragment-bound pockets remains a much more difficult problem [14] as it requires a searchable archive of fragment-bound subpockets [1517] and a computational method focusing on local subpocket descriptors. Consequently, there are still very few reports of experimentally verified subpocket similarity examples that have enabled the transfer of chemical fragments across unrelated proteins [18]. To fill the need for local similarity searching methods while comparing pockets of different sizes, we developed a novel method (ProCare) [17] relying on point 
cloud registration, a numerical image processing to find a spatial transformation (e.g., scaling, rotation and translation) that aligns two point clouds $[19,20]$. ProCare uses as input a point cloud representation of the protein pocket or subpockets, where each point is annotated by eight possible pharmacophoric features (hydrophobic, aromatic, h-bond donor, h-bond acceptor, h-bond donor and acceptor, positive, negative, dummy) complementary to that of the pocket microenvironment [21]. Since ProCare uses local descriptors to compare and align binding subpockets, the method is particularly suited to fragment-based design strategies aimed at positioning fragments in subpockets of any druggable cavity.

While validating the method by focused benchmarking studies [17], we noticed some unexpected local similarity between subpockets from two unrelated proteins with $23 \%$ sequence identity: human tumor necrosis factor alpha (TNF- $\alpha$ ) trimer [22] and human immunodeficiency virus type 1 reverse transcriptase (HIV-1 RT) [23]. On the one hand side, TNF- $\alpha$ is a homotrimeric pro-inflammatory cytokine involved in autoimmune disorders such as rheumatoid arthritis and Crohn's disease [24]. It is currently targeted by monoclonal antibodies preventing its recognition by TNF- $\alpha$ receptors (TNFR1 and TNFR2). To date, no small molecule TNF- $\alpha$ inhibitor has reached the market [22]. On the other side, HIV-1 RT is an enzyme used by the HIV virus to replicate its genome by first generating a complementary DNA from the viral RNA template. HIV-1 RT can be blocked by many marketed drugs [25] binding to either the catalytic site (nucleoside inhibitors, e.g. zidovudine) or a remote allosteric pocket (non-nucleoside inhibitors, e.g. efavirenz).

To exclude potential artifacts or biases and provide a strong statistical support to this initial prediction, we here systematically compared the inner cavity of three inhibitor-bound TNF- $\alpha$ trimer structures with 122 non-nucleoside inhibitor-bound HIV-1 RT X-ray structures. In a large majority of pairwise comparisons, the corresponding subpockets were deemed similar, a prediction that could be confirmed by biophysical experiments evidencing a direct micromolar binding of non-nucleoside HIV-1 RT inhibitors to human soluble TNF- $\alpha$. Interestingly, this unexpected similarity could not be recovered by state-of-the-art cavity 
8

comparisons tools suggesting the unique ability of ProCare to delineate subtle local relationships between unrelated target cavities.

\section{RESULTS AND DISCUSSION}

Identifying similarity between pockets from different proteins suggests that the latter might bind to similar molecules. Although molecular recognition is a dynamic and complex process, the above hypothesis is worth investigating in drug design for hit discovery or for potential off-targets detection. We previously described ProCare [17], a novel computational method relying on a point cloud registration algorithm [19, 20] to assess the similarity between protein pockets. ProCare computes and uses local descriptors, which makes it particularly suitable for detecting local similarities among cavities of different sizes. Typically, ProCare aligns the cavities, described by a cloud of 3D points labeled with pharmacophoric features, by comparing the point descriptors and then derives a similarity score. While benchmarking the ProCare method by comparing protein structures disclosed for the first time to 31,570 subpockets from the sc-PDB archive [8] (Figure 1), we noticed unexpected high similarities between the core pocket at the interface of an inhibitor-bound asymmetric human TNF- $\alpha$ trimer (PDB ID 6OOY) [22], and several non-nucleoside binding sites of inhibitor-bound HIV-1 RT (Supporting Table S1). Computing the area under the receiveroperating characteristic (ROCAUC) curves (Supporting Figure S1) from pairwise ProCare similarity score distributions, the selected non-nucleoside HIV-1 RT subpockets are statistically similar to the inner core of the TNF- $\alpha$ trimer with ROCAUC values higher than 0.80 , a remote similarity not being observed by five other randomly selected targets (Table 1). 


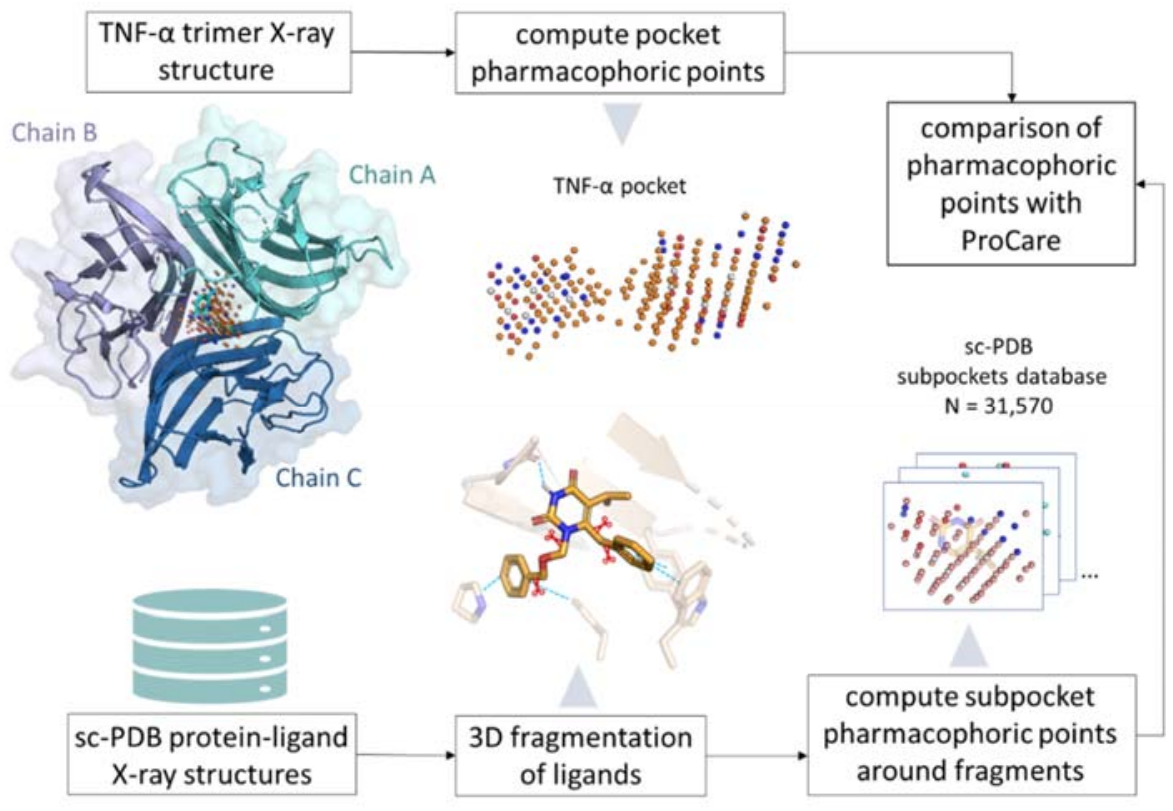

101 Fig. 1 Virtual screening of sc-PDB subpockets for similarity to the core cavity TNF- $\alpha$. The inner pocket of TNF- $\alpha$ (PDB ID 6OOY) is converted as a cloud of points with pharmacophoric properties (orange: hydrophobic, green: aromatic, blue: H-bond donor and positive ionisable, red: h-bond acceptor and negative ionisable, white: dummy) and compared to the corresponding point clouds originating from fragment-bound subpockets of Sc-PDB ligands.

Table 1 Area under the ROC curve of pairwise ProCare similarity scores. ${ }^{a}$

\begin{tabular}{|c|c|c|c|}
\hline Target & Site & Number of subpockets ${ }^{b}$ & ROCAUC \\
\hline HIV-1 RT & non-nucleoside & $195(122)$ & 0.84 \\
\hline$\beta 2$ adrenergic receptor & orthosteric & $14(14)$ & 0.35 \\
\hline Carbonic anhydrase II & catalytic & $183(137)$ & 0.38 \\
\hline Cyclin-dependent kinase 2 & catalytic & $461(274)$ & 0.63 \\
\hline Heat shock protein $90 \alpha$ & catalytic & $214(117)$ & 0.64 \\
\hline Thrombin & catalytic & $253(126)$ & 0.35 \\
\hline
\end{tabular}


111 To further investigate that hypothesis, exclude a potential bias in the ProCare alignment/scoring method

112 and give a stronger statistical support to our prediction, we systematically compared all binding sites from

113 available asymmetric human TNF- $\alpha$ X-ray structures [22] to that of 122 HIV-1 RT structures bound to non-

114 nucleoside inhibitors.

116 Exhaustive comparison of TNF- $\alpha$ trimer and HIV-1 reverse transcriptase binding sites. A ProCare

117 similarity matrix was built by comparing cavities of three asymmetric TNF- $\alpha$ structures (PDB identifiers

118 6OOY, 6OOZ and 6OP0) co-crystallized with benzimidazole inhibitors to 122 cavities (Supporting Table S2)

119 of non-nucleoside HIV-1 RT inhibitors (Figure 2). We observed that $76 \%$ of all pairwise comparisons were

120 scored higher than the previously statistically determined ProCare similarity score threshold of 0.47 in a

121 previous study [17] (Figure 2A). To exclude the possibility that the predicted similarity is caused by peculiar

122 mutations of the HIV-1 RT non-nucleoside biding site, we also compared pairwise similarities for both wild

123 type and mutated HIV-1 RT pockets, but did not observe significant differences in the percentage of HIV-

1241 RT pockets predicted similar to that of TNF- $\alpha$ ( $74 \%$ and $82 \%$ of similar pockets for wild type and mutants,

125 respectively). We thus conclude that the predicted similarity between pockets from these two unrelated

126 targets is independent on the chosen PDB structures and is not biased by mutations in the HIV-1 RT binding

127 site. 

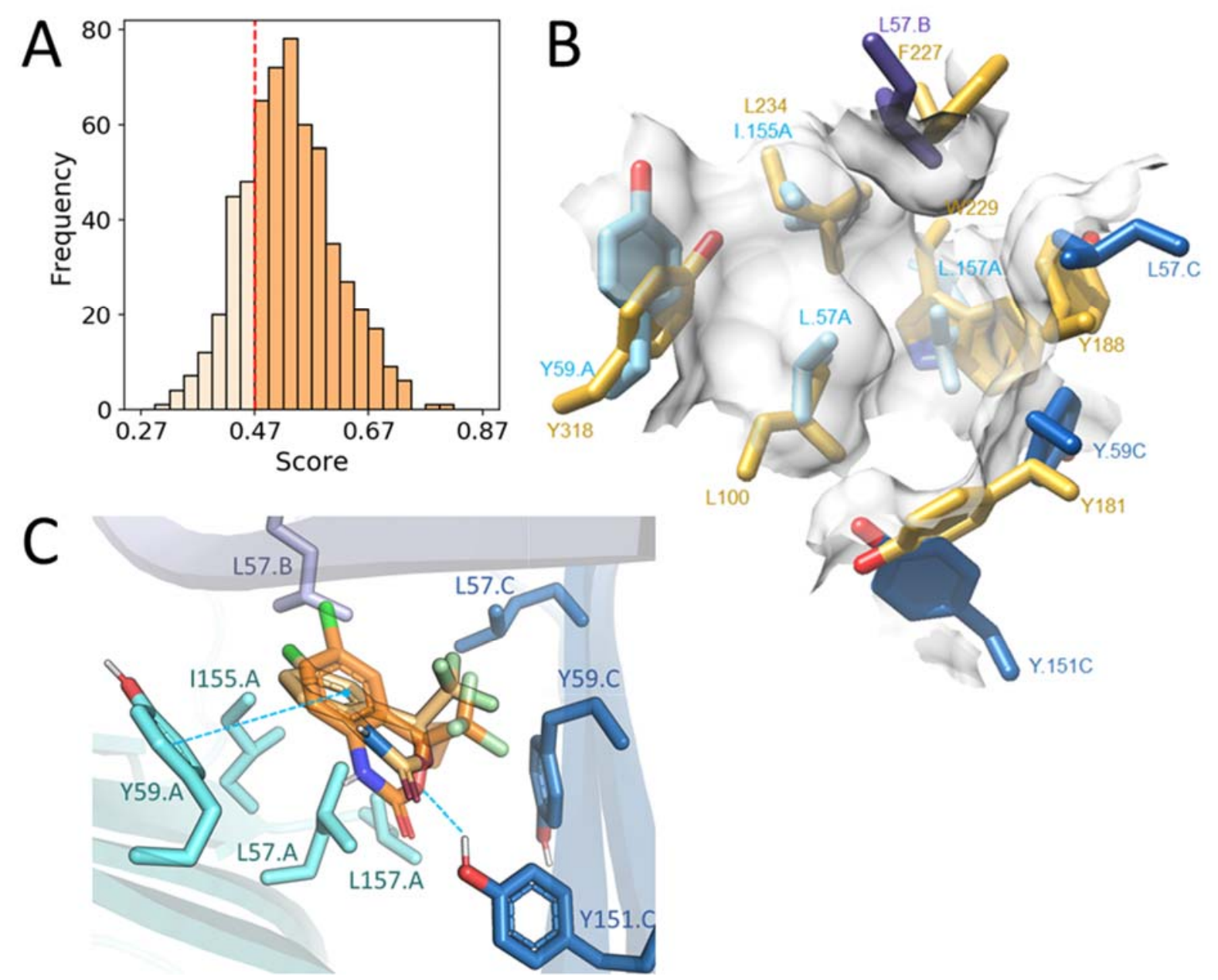

129 Fig. 2 Comparison of TNF- $\alpha$ trimer and HIV-1 RT binding sites with ProCare.

130 (A) Distribution of pairwise similarity scores. Entries scoring above 0.47 ( $p$-value=0.05; threshold marked by the red dashed line) are considered similar according to a previous statistical analysis of 2 million pairwise alignments [17]. (B) Aligned residues of TNF- $\alpha$ (chain A: cyan, chain B: dark slate blue, chain C: cornflower blue; PDB code: 6OOZ) to HIV-1 RT (orange, PDB code: 1FKO) after rotation and translation of HIV-1 RT protein with the resulting ProCare alignment matrix. The surface of the TNF- $\alpha$ inner pocket is depicted in white. (C) ProCare alignment of efavirenz main fragment (light orange) in the TNF- $\alpha$ trimer pocket and PLANTS docking (transparent orange) in the TNF- $\alpha$ trimer pocket (PDB code: 6OOZ). Edge-toface aromatic interaction with TYR59 of TNF- $\alpha$ chain A and hydrogen bond with TYR151 of TNF- $\alpha$ chain C are depicted by blue dashed lines.

Since ProCare yields a transformation matrix to align the compared objects (subpockets onto the target pockets), we herein provided the visual analysis for one entry (efavirenz-bound subpocket) aligned to the TNF- $\alpha$ structure 600Z. Pairs of residues of equivalent interaction properties (aromatic, hydrogen bond 
donor and acceptor, hydrophobic) respectively in TNF- $\alpha$ and HIV-1 RT were nicely matched (Figure 2B)

144 demonstrating that the similarity caught with the point clouds is truly present at the residue level.

145 Matched TNF- $\alpha$ /HIV-1 RT residues were: LEU57.A/LEU100; TYR59.A/TYR318; ILE155/LEU234;

146 LEU157/TRP229; LEU57.B/PHE227; LEU57.C/TYR188; TYR59.C/TYR181 and TYR151.C/TYR181. Inspection

147 of the matched pharmacophoric points that are contributing to the ProCare score showed a mixed

148 contribution of aromatic, hydrogen bond donor and hydrophobic points (Supporting Figure S2) in

149 agreement with the aligned residues (Figure 2B) and the statistics of the contributions of the eight

150 pharmacophoric features to the detected similarity (Supporting Figure S3). Furthermore, efavirenz was

151 docked into TNF- $\alpha$ binding site $600 Z$ with PLANTS [26] after validation of the docking protocol by self-

152 docking of the cocrystalized ligand UCB-5307 in $600 Z$ (RMSD of top-ranked pose by ChemPLP to crystal

153 coordinates: $0.47 \AA$ ). The ProCare-aligned efavirenz fragment in TNF- $\alpha$ fitted well with one of the PLANTS

154 docking solutions (ranked $3^{\text {rd }} / 10$ with a ChemPLP score of -79.32 ), corresponding to a RMSD of $1.8 \AA$

155 (Figure 2C). Aside the potential hydrophobic interactions in the TNF- $\alpha$ binding site, efavirenz docking pose

156 displayed an edge-to-face aromatic interaction with residue TYR59.A and a hydrogen bond with TYR151.C.

157 Interestingly, efavirenz bound to HIV-1 RT protein structure (1FKO) exhibits an edge-to-face aromatic

158 interaction with residue TYR318 [27] (Supporting Figure S4A) that was matched by ProCare to TYR59.A in

159 TNF- $\alpha$ (Figure 2B). Both TYR59.A and TYR151.C are key residues [22] involved in the micromolar and

160 nanomolar binding of the co-crystalized ligands UCB-6876, UCB-5307 and UCB-9260 (Figure 3) in the TNF-

$161 \alpha$ structures 600Y, 600Z, 60P0; the interaction between TYR151.C residue and the benzimidazole moiety

162 being a hydrogen bond (Supporting Figure S4B). Altogether, these observations are strongly suggesting

163 that subpockets in the non-nucleoside binding site of HIV-1 RT are similar to the TNF- $\alpha$ trimer cavity. 
A

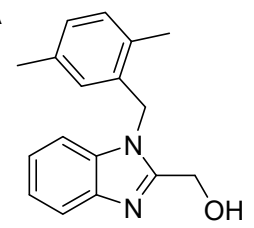

UCB-6786 (6OOY)

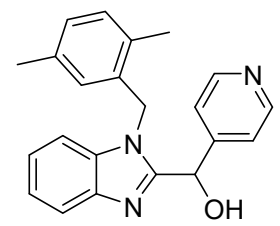

UCB-5307 (6OOZ)

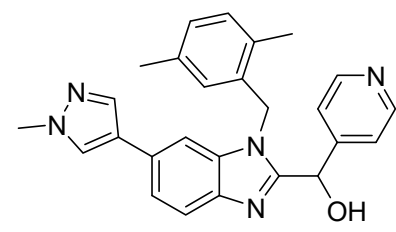

UCB-9260 (6OP0)
B<smiles>O=C1c2ccccc2C(=O)N1CCOC(=S)Nc1ccc(I)cc1</smiles>

Q27097507 (2VG7)<smiles>CC(C)c1c(Cc2ccccc2)n(COCc2ccccc2)c(=O)[nH]c1=O</smiles>

TNK-651 (1S1V)<smiles>CC(F)(C#CC1CC1)c1c(F)ccc(Cl)c1OC(=O)Nc1ccc(Cl)cc1</smiles>

Efavirenz (1FKO)

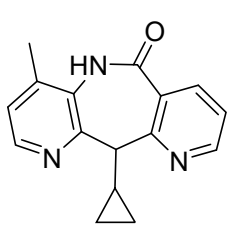

Nevirapine (1LWC)
164

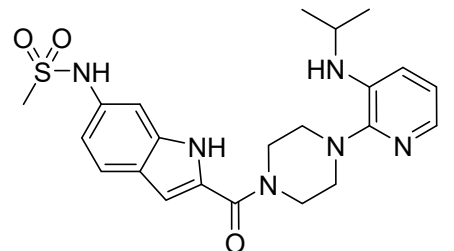

Delavirdine (1KLM)

Fig. 3 Structures of TNF- $\alpha$ and HIV-1 RT non-nucleoside inhibitors. (A) TNF- $\alpha$ inhibitors and (B) HIV-1 RT non-nucleoside inhibitors (PDB entries between brackets).

Assuming that similar binding sites should accommodate similar ligands, HIV-1 RT non-nucleoside inhibitors should therefore bind to TNF- $\alpha$. In order to prioritize HIV-1 RT inhibitors for experimental validation of our hypothesis, we checked which inhibitors were bound to the HIV-RT subpockets that are predicted by ProCare as the most similar to the TNF- $\alpha$ cavity (Table 2).

Among the corresponding inhibitors, two easily purchasable FDA-approved drugs (efavirenz, neviparine; Figure 3) are almost entirely buried in the HIV-1 RT subpockets found similar to the TNF- $\alpha$ cavity, exhibit a size and molecular volume similar to that of two TNF- $\alpha$ inhibitors (UCB-6876 and UCB-5307; Figure 3) and were therefore selected for biological evaluation. In addition, we also considered a third marketed inhibitor (delavirdine; Table 2, Figure 3) whose pocket was found much less similar to that of TNF- $\alpha$, although just above the 0.47 ProCare similarity threshold. 
178 Table 2 Bound inhibitors of the HIV-1 reverse transcriptase cavities found similar to TNF- $\alpha$ cavities

\begin{tabular}{lllll}
\hline HIV-RT Inhibitor $^{\text {a }}$ & HIV1-RT PDB entry & TNF- $\alpha$ PDB entry & ProCare score & Rank \\
\hline Q27097507 & 2VG7 & $600 Z$ & 0.810 & 1 \\
Efavirenz & 1FKO & $600 Z$ & 0.773 & 2 \\
Nevirapine & 1LWC & $600 Z$ & 0.737 & 3 \\
TNK-651 & 1S1V & $600 Z$ & 0.731 & 4 \\
Nevirapine & 2HNY & $600 Z$ & 0.729 & 5 \\
$\ldots$ & & $\ldots$ & $\ldots$ & $\ldots$ \\
Delavirdine $^{\text {b }}$ & 1KLM & $600 Z$ & 0.484 & 408
\end{tabular}

$179 \quad$ a PDB chemical component identifier (Drug name in brackets).

$180{ }^{b}$ After manual fragmentation, a higher ProCare score (0.599) was obtained for the subpocket of

181 delavirdine's fragment \#2 (Supporting Figure S5) against 600Y pocket (Supporting Table S3).

Non-nucleoside HIV-1 RT inhibitors bind to human TNF- $\alpha$. Three different non-nucleoside FDA-approved drugs (nevirapine, efavirenz and delavirdine) were tested for direct binding to a fluorescent-labelled TNF$\alpha$ trimer by microscale thermophoresis (MST), a robust and sensitive biophysical method to detect and quantify molecular interactions in solution $[28,29]$. The MST signal is based on ligand-dependent temperature-induced changes (thermophoresis, temperature-related fluorescence intensity) of the fluorescence emission of the labelled protein target. The $17.3 \mathrm{kDa}$ homotrimeric TNF- $\alpha$ that spontaneously assemble in solution $[30,31]$ was therefore labelled by a RED-fluorescent probe for MST experiments in presence of increasing concentrations of the three HIV-1 RT inhibitors (Figure 4). MST traces in presence of efavirenz and delavirdine showed distinct states (from bound to unbound), indicating a direct interaction of these two inhibitors with TNF- $\alpha$ (Figure 4A, B). Dissociation constants $\left(K_{D}\right)$ 

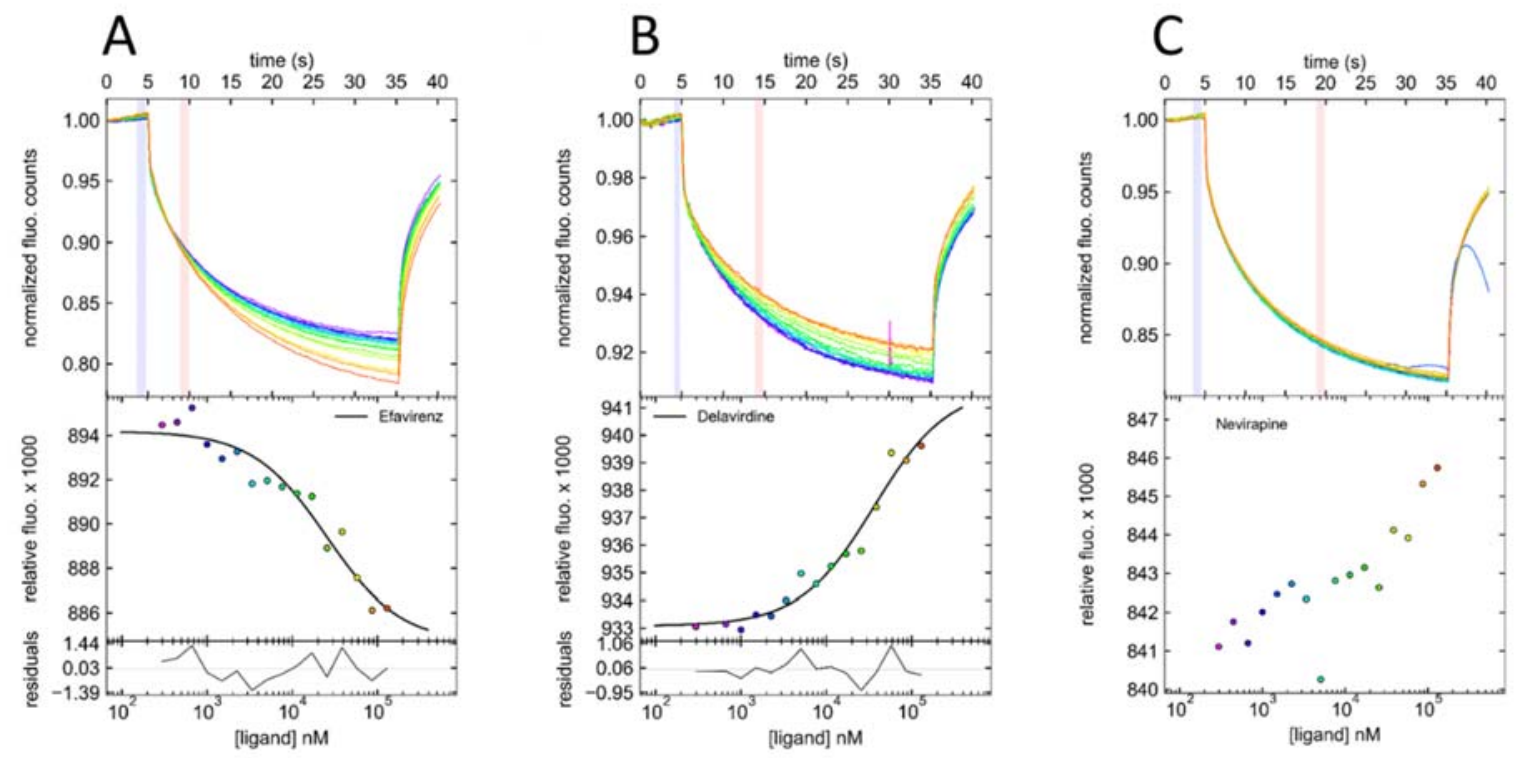

Fig. 4 Microscale thermophoresis (MST) demonstrates a direct interaction between HIV-1 RT inhibitors and RED fluorescent-tagged TNF- $\alpha$.For analysis, the change in thermophoresis is expressed as the change in the normalized fluorescence $\left(\Delta F_{\text {norm }}\right)$, which is defined as $F_{\text {hot }} / F_{\text {cold }}(F$-values correspond to average fluorescence values between defined areas marked by the red and blue cursors, respectively). Titration of the non-fluorescent ligand results in a gradual change in thermophoresis, which is plotted as $\Delta F_{\text {norm }}$ to yield a binding curve, which can be fitted to derive binding constants. (A) Experimental MST traces of efavirenz $\left(K_{\mathrm{D}}=24 \pm 8 \mu \mathrm{M}\right)$; (B) Experimental MST traces of delavirdine $\left(K_{\mathrm{D}}=39 \pm 9 \mu \mathrm{M}\right)$; (C) Experimental MST traces of nevirapine. Only the best MST traces (highest signal to noise ratio) are shown here. Values for all experiments conducted according to different experimental protocols are listed in Supporting Table S4. observations were insensitive to experimental protocols (buffer composition, solubilizing agents, incubation time, MST power; Supporting Table S4). 
212 In absence of X-ray structures of TNF- $\alpha$ bound to efavirenz and delavirdine, we cannot rule out the 213 possibility that both inhibitors bind to a different pocket than that highlighted in the current computational 214 study. This hypothesis is however very unlikely for two reasons: (i) no other cavity than that occurring at 215 the inner core of the multimeric TNF- $\alpha$ could be detected among the currently existing 33 structures 216 available in the Protein Data Bank; (ii) all non-covalent small molecular weight inhibitors co-crystallized 217 with TNF- $\alpha$ dimeric or trimeric forms [32-35] are exactly bound at the central pocket examined in this 218 study.

219 We should recall here that none of the HIV-1 RT inhibitors has been optimized for binding to TNF- $\alpha$ and 220 directly repurposable for treating TNF- $\alpha$-dependent autoimmune disorders. However, the recently 221 discovered UCB6786 TNF- $\alpha$ inhibitor (Figure 3) [22], a fragment hit with a ligand efficiency similar to that 222 of efavirenz (UCB6786: $0.34 \mathrm{kcal} / \mathrm{mol}$ per non-hydrogen atom; efavirenz: $0.32 \mathrm{kcal} / \mathrm{mol}$ per non-hydrogen 223 atom) could be easily optimized to yield the $9 \mathrm{nM}$ inhibitor UCB-5307 (Figure 3) by just occupying a side 224 pocket formed by the three TYR199 side chains of the TNF- $\alpha$ homotrimer with a pyridyl ring [22]. Structure225 guided efavirenz optimization for TNF- $\alpha$ binding is therefore possible by appropriate trimming of unnecessary cyclopropylethynyl substituent and occupation of the above-described potency subpocket.

The similarity between TNF- $\alpha$ trimer and HIV reverse transcriptase binding sites is not obvious. To demonstrate whether the herein disclosed similarity between the human TNF- $\alpha$ trimer and the HIV-1 RT non-nucleoside binding sites is obvious, we performed the same set of pairwise binding site comparisons, as that previously reported for ProCare (Figure 2), with state-of-the-art methods [10] developed either inhouse (FuzCav [36], Shaper [21] and SiteAlign [37]) or by third parties (G-LoSA [38], KRIPO [15] and ProBiS

233 [39]). The binding site perception, comparison algorithm and scoring function is specific to each method.

234 Some methods (FuzCav, SiteAlign) consider entire cavities while some others utilize either fragment-bound 235 subpockets (KRIPO, Shaper) or local protein descriptors (G-LoSA). To make the comparison consistent, the 
same set of atomic coordinates were compared, a binding site being defined by the protein PDB identifier,

237 the ligand HET code, chain identifiers and list of amino acids lining the cavity. The only exception was for

238 the KRIPO method, which used all the chains available in the PDB entry, but still corresponding to the same

239 tuple (PDB, HET) as for the other methods. For each method, the distribution (Figure 5) and percentage of

240 pairwise comparisons scored above the developer's recommended similarity threshold (Table 3) were

241 reported.

242 Table 3 Comparison of three TNF- $\alpha$ and 122 HIV-1 RT non-nucleoside binding sites by state-of-the-art 243 cavity comparison methods.

\begin{tabular}{lllc}
\hline Method & Score threshold & Metric & Success rate $^{\mathbf{b}}$ \\
\hline G-LoSA & 0.59 & GA-score & 35.2 \\
KRIPO & 0.50 & Modified Tanimoto coefficient & 5.8 \\
Shaper & 0.44 & ColorRefTversky & 1.4 \\
SiteAlign & $0.6,0.2$ & $\mathrm{~d} 1$ and d2 distances & \\
FuzCav & 0.16 & Tanimoto coefficient & 0.3 \\
ProBiS & 2 & Z-score & 0 \\
ProCare & 0.47 & ProCare score & 0 \\
\hline
\end{tabular}

${ }^{a}$ Developer's recommended similarity/distance threshold for estimating two binding sites similar

$245{ }^{b}$ Percentage of pairwise comparisons scored above the threshold.

$246{ }^{\mathrm{C}}$ For SiteAlign comparisons, pairs are considered similar when the two distances $(\mathrm{d} 1, \mathrm{~d} 2)$ are below an 247 acceptable cut-off.[37]

$248{ }^{d}$ The Z-score indicates the statistical relevance of ProBiS binding site alignments.

Strikingly, only the G-LoSA method relying on a graph-based local alignment of cavity-lining amino acids, managed to find some similarity between the two sets of binding sites, however with reduced success rate

252 (35.2\%) when compared to the ProCare algorithm (76.6\% success rate; Table 3). We acknowledge that the developer's recommended thresholds may be biased toward peculiar datasets. However, all methods 

each pairwise comparison.
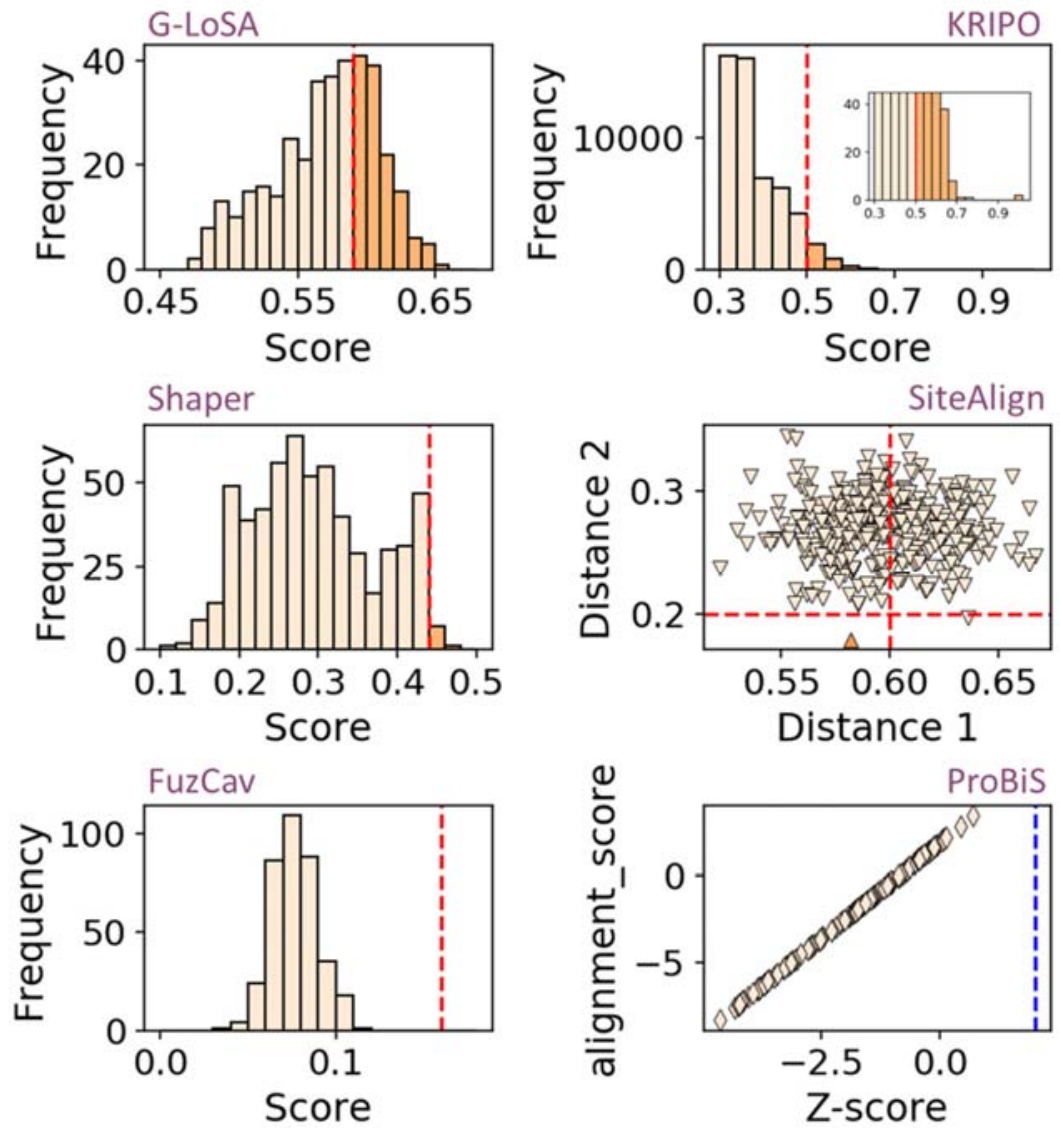

Fig. 5 Score distribution of pairwise comparisons between binding sites of TNF- $\alpha$ trimer and HIV-1 reverse transcriptase. Binding sites in asymmetric structures of TNF- $\alpha$ trimer $(n=3)$ were compared to binding sites of non-nucleoside inhibitors in HIV-1 reverse transcriptase (sc-PDB set, $n=122$ ). Pairs with similarity measures scored above each method-specific threshold (red dashed line) were considered similar. For SiteAlign comparisons, pairs are considered similar in case the two distances (distance 1, distance2) are below the recommended cut-off. For ProBiS, the threshold above which an alignment is considered significant is marked by the blue dashed line.

The herein reported binding of some HIV-1 RT non-nucleoside inhibitors to human TNF- $\alpha$ remains unobvious to many binding site comparison algorithms. Would this unexpected feature be better captured by remote ligand similarities? To investigate this question, we compared 2D and 3D descriptors of the corresponding inhibitors (Figure 6). 

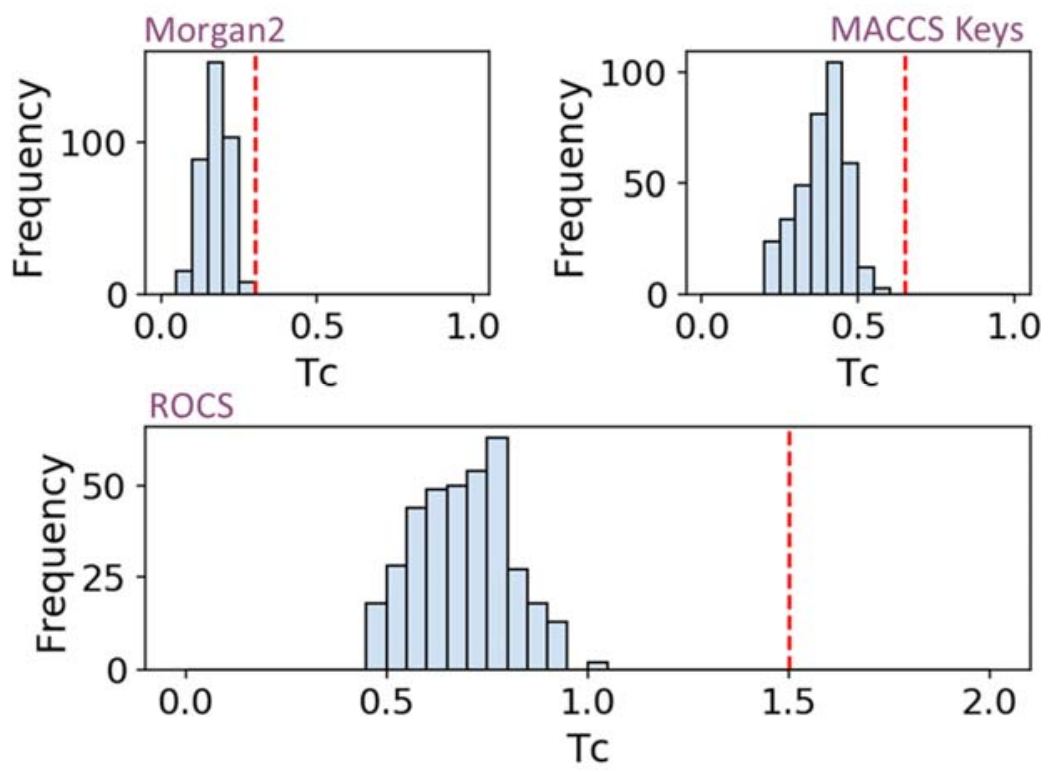

Neither comparing 2D fingerprints nor 3D shapes would have confidently suggested possible binding of HIV-1 RT inhibitors to TNF- $\alpha$ trimer (Figure 6) since none of the considered ligand pairs exhibit a pairwise similarity above an acceptable threshold (Morgan2 circular fingerprint: 0.30 [40]; 166 public MACCS keys: 0.65 [40], TanimotoCombo ROCS 3D similarity: $1.5[41,42])$. We should precise here that 3D similarities were inferred from PDB protein-bound ligand X-ray structures and that alternative conformations might be selected by the two targets, although the very rigid efavirenz does indeed bind to the two proteins of interest albeit with different affinities. Extending 2D fingerprint comparisons to additional 2,361 HIV-1 RT inhibitors (Supporting Table S5) from the ChEMBL database [43], did not change our conclusion since only keys, respectively (data not shown). 


\section{CONCLUSION}

Herein, we describe a systematic comparison of fragment-bound subpockets from a priori unrelated targets (TNF- $\alpha$, HIV-1 RT) but predicted to share local similarities according to our recently-developed ProCare point cloud registration method. The computational prediction was verified by microscale thermophoresis experiments evidencing the micromolar binding of some but not all HIV-1 RT nonnucleoside inhibitors to human soluble TNF- $\alpha$. Interestingly, the ProCare prediction could not be revealed by other state-of-the-art cavity or ligand similarity search methods. Point cloud registration, a computational method frequently used for digital image processing in robotics and medical imaging, enables the detection of subtle and local protein similarities thanks to a powerful description of subpocket microenvironments. The ProCare method appears as a promising idea generator for drug repurposing and fragment-based ligand design since it is able to pick starting ligands at a proteomic scale.

\section{METHODS}

TNF- $\alpha$ structures. The recently described asymmetric structures of the human TNF- $\alpha$ trimer bound to different inhibitors were retrieved from the RCSB Protein Data Bank (PDB) homepage (https://www.rcsb.org) [44] using the following identifiers: 600Y, 60P0, 600Z [22]. The PDB structures were protonated with Protoss [45] v.4.0, then split into protein, ligands and water molecules and finally converted into mol2 format with Sybyl-X v.2.1.1 (Certara USA, Inc., Princeton, NJ 08540). The binding sites ('SITE') were defined as any protein residue with at least one heavy atom closer than $6.5 \AA$ from any ligand heavy atom and saved in mol2 and pdb formats. The ligands were converted into sdf format with OpenEye Python toolkits v.2020.0.4 (OpenEye Scientific Software, Santa Fe, U.S.A.). Cavities were detected with IChem v.5.2.9 VolSite utility [21] (cavity_all output) using default parameters. 
312 sc-PDB fragments-subpockets. Ligands coordinates from the sc-PDB (http://bioinfo-pharma.u-

313 strasbg.fr/scPDB) [46] v.2016 archive were fragmented in 3D with the IChem v.5.2.9 fragmentation utility

314 [47]. Fragmentations occurs in the binding sites so that only the main fragments interacting sufficiently

315 (four interactions of which at least one is polar) with their target proteins were kept. Finally, the cavity

316 pharmacophoric points cloud were computed at $4 \AA$ from the fragments center to describe the protein

317 subpocket, using the IChem v.5.2.9 VolSite utility (cavity_4 output). The cavity points are labeled with eight

318 possible pharmacophoric features (hydrophobic, aromatic, h-bond donor, $\mathrm{h}$-bond acceptor, $\mathrm{h}$-bond donor

319 and acceptor, positive, negative, dummy) that are complementary to the features of the nearest protein

320 atom. Cavities with less than three points were discarded. A total of 31,570 valid fragment-bound

321 subpockets was finally obtained.

323 Virtual screening of sc-PDB subpockets. All sc-PDB subpockets $(n=31,570)$ were compared to TNF- $\alpha$

324 pockets with ProCare v.0.1.0. using default parameters and scoring scheme. ProCare is based on a point

325 cloud registration algorithm that first computes a local descriptor for each pharmacophoric point, that

326 account for the shape and the pharmacophoric features distribution around that point. Good matches of

327 areas in the two compared cavities are searched for with a random sampling consensus and the derived

328 alignment is refined iteratively. A match of two points is scored positively when they are within $1.5 \AA$ to

329 each other and have the same pharmacophoric feature. The screening was evaluated by computing the

330 receiver operating characteristics ROC curve and its area under the curve AUC considering the HIV-1 RT

331 entries as positives and any other entries as negatives. The same ROC AUC calculation was repeated, each

332 time considering the following human targets as positives, any other entry being the negatives: beta-2 

90-alpha.

Docking. TNF- $\alpha$ x-ray structure $600 Z$ was prepared as described above (see TNF- $\alpha$ structures). 600Z cocrystallized ligand on the one hand, delavirdine, efavirenz and nevirapine as well as their main fragments on the other hand were drawn with MarvinSketch v.16.10.17 (ChemAxon Ltd, 1031 Budapest, Hungary) and saved into 2D sdf format. They were ionized with Filter v.2.5.1.4 (OpenEye Scientific Software, Santa Fe, U.S.A.) using customized parameters (Supporting Table S6). Then Corina v.3.40 (Molecular Networks GmbH, 90411 Nürnberg, Germany) was used to generate a starting 3D conformation for each inhibitor. The prepared molecules were docked into the target $600 Z$ with PLANTS v.1.2 [26] using the following configuration: the grid was set at $13 \AA$ from the binding site center; poses were searched 'speed1' settings to generate a maximum of 10 poses per ligand using a clustering rmsd of $2 \AA$. Solutions were scored with

345 the default ChemPLP scoring function [26]. The docking protocol was validated by computing the rmsd 346 between of the docked $600 Z$ ligand coordinates and the x-ray coordinates. Results were processed and 347 rescored by computing the interaction fingerprint (IFP) similarity (Tanimoto metric) [48] between X-ray 348 and docking poses. The IFPs were computed with IChem v.5.2.9 IFP module. All poses were visually 349 inspected using Maestro v.2019-3 (Schrödinger, New York, NY 10036-4041).

HIV-1 reverse transcriptase PDB structures. Starting from the PDB structure 1VRT as a reference, a search was performed in the RCSB PDB (https://www.rcsb.org) [44] to retrieve all structures with strict matching

353 ("Structure Similarity" query in the PDB). After visual check, 122 entries already available in the sc-PDB repository (http://bioinfo-pharma.u-strasbg.fr/scPDB) [8] and for which the ligand is a non-nucleoside 
PDB identifiers and Uniprot accession numbers is reported Supporting Table S2. According to the sc-PDB

357 preparation rules, the binding sites ('SITE') were defined as described above. Protein, ligand and binding

358 site 'SITE' structures were directly retrieved in mol2 file format from the Sc-PDB archive. The corresponding

359122 ligands were 3D-fragmented with the IChem v.5.2.9 [49] fragmentation utility [47] and the 360 complementary VolSite [21] cavity points, computed at $4 \AA$ around each fragment were finally saved.

HIV-1 reverse transcriptase ChEMBL ligands. Bioassay information were retrieved from the ChEMBL [43] dataset (Release 28; https://www.ebi.ac.uk/chembl) by querying the general keyword 'reverse transcriptase' and retaining ChEMBL target identifiers (CHEMBL247, CHEMBL4296301, CHEMBL2366516) corresponding to HIV-1 RT. Ligands with a measured sub-micromolar half-maximal inhibitory concentration $\left(\mathrm{IC}_{50}\right)$ against the HIV1-RT single target were defined here as inhibitors (Supporting Table S5). The corresponding SMILES strings were retrieved and further processed with RDKit (Open-source cheminformatics; http://www.rdkit.org) v.2019.03.4.0 to remove redundancy.

\section{Binding sites comparisons.}

ProCare. ProCare [17] v.0.1.1 pairwise comparison were performed on cavities computed with the VolSite module [21] in IChem v5.2.9 [49]. Entire cavities ("cavity_all" output) were calculated for TNF- $\alpha$ structures whereas only cavity points closer than 4.0 A from any fragmented ligand heavy atom ("cavity_4" output) were considered for HIV-1 RT binding sites. VolSite cavity points were directly used for point cloud registration and determination of colored fast point feature histograms (c-FPFH) as previously described

376 [17]. Finally, the respective set of c-FPFH descriptors for the two cavities were aligned and compared to 377 each other using a RANSAC algorithm $[19,20]$ with default parameters [17]. Alignments results were 378 scored with the default ProCare scoring function [17] which evaluates with a Tversky metric the proportion 
of aligned points of the same pharmacophoric features. In agreement with our previous study [17] where the similarity threshold of 0.47 ( $p$-value of 0.05 ) was statistically determined, pockets scoring above 0.47 were considered similar.

FuzCav. FuzCav [36], an alignment-free method, was used to compare the binding site 'SITE' (mol2 format) entries of TNF- $\alpha$ dataset to the binding sites of HIV-1 RT sc-PDB dataset. Each binding site was tagged to compute a 4,833 bit-string that count all possible pharmacophoric triplets based on the atomic coordinates of $\mathrm{C} \alpha$ atoms lining the binding cavity. The pairwise comparisons of the fingerprints were evaluated with the default similarity score, with a threshold set at a value of 0.16 to distinguish similar from dissimilar binding sites.

G-LoSA. G-LoSA [38] v.2.2 is an alignment tool that was used with the binding sites 'SITE' pdb files. G-LoSA computes a set of inter-structural $\mathrm{C} \alpha$ pair distances to derive a graph, which will later be subjected to maximum clique search. The default G-LoSA score (GA-score) was used to evaluate the alignments. A threshold value of 0.59 , recommended by the authors [38] and corresponding to a p-value of 0.05 , was used to distinguish similar from dissimilar binding sites.

KRIPO. PDB ligands structural information were downloaded from Ligand Expo (http://ligandexpo.rcsb.org/) and prepared according to the KRIPO procedure (https://github.com/3D-e-Chem/kripo). Then KRIPO [15] v.1.0.1 was used with the list of prepared PDB structures for the pharmacophore fuzzy fingerprints calculations, using default parameters (fragmentation procedure activated). The pairwise similarities of the fingerprints were estimated with kripodb (v.3.0.0) using the modified Tanimoto 
Shaper. Shaper [21] v.1.0 uses the same input files (VolSite cavities in mol2 file format) as ProCare. Shaper

421 is an alignment method based on the OpenEye ShapeTK toolkit (OpenEye Toolkits 2020.2.0, OpenEye 422 Scientific Software, Santa Fe) to maximize the overlap of shape and pharmacophoric features of the two

ProBiS. In a first place, the surface information (srf files) was computed for each prepared PDB structures with the default parameters referenced in the manual (3.0 $\AA$ to the ligand). ProBiS [39] requires a list of ligand HET code and residue number for each PDB entries. That list was provided to ensure that the ligands/sites considered are the same as in the binding site datasets used for other methods. Then, the alignment and comparison of the srf files were executed with default parameters, except for the Z-score that was set to a high negative value (-9999) as suggested by the authors to enforce the output of all results. Similarity between two binding sites was evaluated by the alignment score and Z- score. A threshold Z-score value of 2.0 was used to distinguish significant from irrelevant binding site alignments.

SiteAlign. For each entry, the list of natural amino acids in the 'SITE' mol2 files were provided as input. SiteAlign [37] v.4.0 describes a binding site by a polyhedron of 80 discretized triangles annotated with eight possible pharmacophoric features projected from cavity-lining C- $\alpha$ atoms. This results in a fingerprint of 640 integers. The pairwise comparisons imply aligning the corresponding polyhedron and computing the $\mathrm{d} 1$ and $\mathrm{d} 2$ distances of the fingerprints. The distance thresholds of $\mathrm{d} 1=0.6$ and $\mathrm{d} 2=0.2$ were applied respectively, to discriminate similar from dissimilar binding sites. 
compared cavities, thanks to a smooth Gaussian function. The alignments were realized with default settings and scored with a Tversky metric putting more weight on the reference cavity (RefTve). A threshold RefTve value of 0.44 ( $p$-value $=0.005)$ was used to distinguish similar from dissimilar binding sites.

Ligand 2D similarity. Morgan fingerprints on the one hand, and 166 public MACSS keys on the other hand were computed on the sc-PDB ligands (sdf format) and ChEMBL ligands (SMILES strings) with RDKit (Opensource cheminformatics; http://www.rdkit.org) python package v.2019.03.4.0 using default parameters (radius $=2 \AA$ for the Morgan fingerprints). The Tanimoto coefficients of the pairwise TNF- $\alpha$ ligands/HIV- 1 RT ligands fingerprints comparison were reported. Cut-off values of 0.30 (Morgan fingerprints) and 0.65 (MACCS keys) were used to discriminate chemically similar from dissimilar ligands.

Ligand 3D similarity. Sc-PDB HIV-1 RT inhibitors were compared to TNF- $\alpha$ inhibitors with OpenEye ROCS v.3.2.0.4 and scored by decreasing Tanimoto similarity metric accounting for both shape and chemical features overlap (TanimotoCombo). A TanimotoCombo cut-off value of 1.5 was used to discriminate chemically similar from dissimilar ligands.

Chemicals and biologicals. Nevirapine (catalog \#S1742), efavirenz (catalog \#S4685) and delavirdine mesylate (catalog \#S6452) were purchased from Selleck Chemicals (https://www.selleckchem.com/). Soluble human TNF- $\alpha$ (catalog \# Z01001) was purchased from GenScript (http://www.genscript.com). 

using the RED-NHS 2nd generation labeling kit (NanoTemper Technologies $\mathrm{GmbH}$ ) using a protein concentration of $10 \mu \mathrm{M}$ and a molar dye-to protein ratio 3:1. A label/protein ratio of 0.4 was determined using photometry at 650 and $280 \mathrm{~nm}$. Compounds efavirenz, delavirdine and nevirapine were initially dissolved in DMSO to afford stock solutions of $10 \mathrm{mM}$. These were then diluted to initial concentrations of $260 \mu \mathrm{M}$ into $20 \mathrm{mM} \mathrm{K}$ phosphate $\mathrm{pH} 7.4,150 \mathrm{mM} \mathrm{NaCl}$ ensuring a final concentration of DMSO of 2.6 \%. These compounds were serially diluted $2: 1$ in buffer $20 \mathrm{mM} \mathrm{K}$ phosphate $\mathrm{pH} 7.4,150 \mathrm{mM} \mathrm{NaCl}, 2.6 \%$ DMSO producing ligand concentrations ranging from $260 \mu \mathrm{M}$ to $594 \mathrm{nM}$ (16 titration points). For MST measurements, each ligand dilution was mixed with 1 volume of fluorescently-labelled TNF $\alpha$ at $680 \mathrm{nM}$ in $20 \mathrm{mM} \mathrm{K}$ phosphate $\mathrm{pH} 7.4,150 \mathrm{mM} \mathrm{NaCl}, 0.02 \%$ Tween-20, which leads to a final concentration of TNF $\alpha$ of $340 \mathrm{nM}$ and final ligand concentrations at half of the ranges above. The final buffer is $20 \mathrm{mM} \mathrm{K}$ phosphate $\mathrm{pH} 7.4,150 \mathrm{mM} \mathrm{NaCl}, 0.01 \%$ Tween-20 and $1.3 \%$ DMSO. After a 15-min incubation at room temperature in the dark, followed by centrifugation at $13,000 \mathrm{~g}$ for $3 \mathrm{~min}$, each solution was filled into Monolith NT Premium capillaries (NanoTemper Technologies GmbH). Thermophoresis was measured at $25^{\circ} \mathrm{C}$ with $40 \%$ LED power and $20,40 \%$ and $80 \%$ MST power using a Monolith NT.115 (NanoTemper Technologies $\mathrm{GmbH}$ ). Data were analyzed in the NT Analysis software version 1.5.41 (NanoTemper

460 Technologies $\mathrm{GmbH}$ ).

2D two-dimension

3D three-dimension 
468 HIV Human Immunodeficiency Virus

471 RANSAC Random Sample Consensus

472 RMSD Root Mean Square Deviation

473 ROC Receiver Operating Characteristics

474 RT Reverse Transcriptase

475 TNF Tumor Necrosis Factor

\section{SUPPLEMENTARY INFORMATION}

479 Figure S1: Receiver operating characteristic (ROC) curves of ProCare similarity scores. Figure S2: ProCare 480 alignment of efavirenz main fragment subpocket onto TNF- $\alpha$ trimer pocket. Figure S3: Contributions of 481 the eight pharmacophoric features to the ProCare similarity score between HIV-1 RT and TNF- $\alpha$. Figure S4: 482 Non-covalent interactions between efavirenz and HIV-1 RT, and between UCB-5307 and TNF- $\alpha$ trimer. 483 Figure S5: Manual fragmentation of delavirdine in three fragments (\#1 to \#3). Table S1: sc-PDB subpockets 484 sorted by decreased ProCare similarity to the inner cavity of human TNF- $\alpha$. Table S2: PDB entries 485 describing non-nucleoside inhibitors bound to HIV-1 reverse transcriptase. Table S3: Comparison of 
delavirdine subpockets, resulting from manual fragmentation, with TNF- $\alpha$ trimer pockets. Table S4: Dissociation constant (KD) of three HIV-1 RT inhibitor binding to human soluble TNF- $\alpha$, according to MST experimental conditions. Table S5: CHEMBL entries describing HIV-1 RT non-nucleoside inhibitors. Table S6: Customized rules for OpenEye Filter ionization.

\section{AVAILABILITY OF DATA AND MATERIALS}

Data. Input and results data are available at https://github.com/kimeguida/ProCare_TNF.

Software. ProCare, version 0.1 .1 and 0.1.0, https://github.com/kimeguida/ProCare; Fuzcav, http://bioinfo-pharma.u-strasbg.fr/labwebsite/downloads/FuzCav.tgz; G-LoSA, version 2.2, https://compbio.lehigh.edu/GLOSA; KRIPODB, version 3.0.0, http://3d-e-chem.github.io/kripodb; KRIPO, version 1.0.1, https://github.com/3D-e-Chem/kripo; ProBiS, http://insilab.org/probis-algorithm/; SiteAlign, version 4.0, http://bioinfo-pharma.u-strasbg.fr/labwebsite/downloads/SiteAlign-4.0.tgz; Shaper, version 1.0, http://bioinfo-pharma.u-strasbg.fr/labwebsite/downloads/Shaper.tgz; RDKit python package, version 2019.03.4.0, https://www.rdkit.org/; ROCS, version 3.2.0.4, https://www.eyesopen.com/rocs; IChem, version 5.2.9, http://bioinfo-pharma.ustrasbg.fr/labwebsite/downloads/IChem_v.5.2.9.tgz: Python OpenEye toolkits version 2020.0.4; FILTER, version 2.5.1.4, https://www.eyesopen.com/filter; PLANTS version 1.2, http://www.tcd.unikonstanz.de/plants_download; Python package Matplotlib version 3.0.2; Maestro vesion 2019-3, https://www.schrodinger.com/products/maestro; Pymol version 2.1, https://pymol.org/2; Sybyl-X v.2.1.1, https://www.certara.com/sybyl-x-software; MarvinSketch version 16.10.17, https://chemaxon.com/products/marvin; 
510 The authors declare no competing interests.

512 FUNDING

513 The authors are thankful to the Doctoral School of Chemical Sciences (EDSC, University of Strasbourg) for 514 a doctoral fellowship to M.E.

517 Conceived and design experiments: ME, DR. Performed the experiments: ME, Analyzed the data: ME, DR. 518 Wrote the paper: ME, DR.

\section{ACKNOWLEDGMENTS}

521 The authors are thankful to the Doctoral School of Chemical Sciences (EDSC, University of Strasbourg) for

522 a doctoral fellowship to M.E. The Calculation Center of the IN2P3 (CNRS, Villeurbanne, France) is

523 acknowledged for the allocation of computing time and excellent support. We sincerely thank Prof. M.

524 Rarey (University of Hamburg, Germany) for providing an executable version of Protoss, OpenEye Scientific

525 Software for the generous allocation of an academic license, and Dr. Catherine Birck (IGBMC, Illkirch) for 526 the microscale thermophoresis experiments. 


\section{AUTHOR INFORMATION}

\section{Corresponding author}

Didier Rognan - Laboratoire d'Innovation Thérapeutique, UMR7200 CNRS-Université de

\section{Author}

Merveille Eguida - Laboratoire d'Innovation Thérapeutique, UMR7200 CNRS-Université de

\section{REFERENCES}

541 1. Sliwoski G, Kothiwale S, Meiler J, Lowe EW (2014) Computational Methods in Drug Discovery. Pharmacol Rev 66:334-395. https://doi.org/10.1124/pr.112.007336

2. Rognan D (2017) The impact of in silico screening in the discovery of novel and safer drug candidates. Pharmacol Ther 175:47-66. https://doi.org/10.1016/j.pharmthera.2017.02.034

3. Ehrt C, Brinkjost T, Koch O (2016) Impact of Binding Site Comparisons on Medicinal Chemistry and Rational Molecular Design. J Med Chem 59:4121-4151. https://doi.org/10.1021/acs.jmedchem.6b00078

548 4. An J, Totrov M, Abagyan R (2004) Comprehensive identification of "druggable" protein ligand binding sites. Genome Inform 15 2:31-41 
550 5. Berman HM (2000) The Protein Data Bank. Nucleic Acids Res 28:235-242.

$551 \quad$ https://doi.org/10.1093/nar/28.1.235

552 6. Bhagavat R, Sankar S, Srinivasan N, Chandra N (2018) An Augmented Pocketome: Detection and Analysis of Small-Molecule Binding Pockets in Proteins of Known 3D Structure. Structure 26:499512.e2. https://doi.org/10.1016/j.str.2018.02.001

7. Kufareva I, llatovskiy A V., Abagyan R (2012) Pocketome: an encyclopedia of small-molecule binding sites in 4D. Nucleic Acids Res 40:D535-D540. https://doi.org/10.1093/nar/gkr825

8. Desaphy J, Bret G, Rognan D, Kellenberger E (2014) sc-PDB: a 3D-database of ligandable binding sites-10 years on. Nucleic Acids Res 43:D399-D404. https://doi.org/10.1093/nar/gku928

9. Pérot S, Sperandio O, Miteva MA, et al (2010) Druggable pockets and binding site centric chemical space: A paradigm shift in drug discovery. Drug Discov Today 15:656-667. https://doi.org/10.1016/j.drudis.2010.05.015

10. Ehrt C, Brinkjost T, Koch O (2018) A benchmark driven guide to binding site comparison: An exhaustive evaluation using tailor-made data sets (ProSPECCTs). PLoS Comput Biol 14:1-50. https://doi.org/10.1371/journal.pcbi.1006483

11. Besnard J, Ruda GF, Setola V, et al (2012) Automated design of ligands to polypharmacological profiles. Nature 492:215-220. https://doi.org/10.1038/nature11691

12. Jenkinson S, Schmidt F, Rosenbrier Ribeiro L, et al (2020) A practical guide to secondary pharmacology in drug discovery. J Pharmacol Toxicol Methods 105:106869. https://doi.org/10.1016/j.vascn.2020.106869 
to find alternative uses of therapeutics. Expert Opin Drug Discov 15:397-401.

https://doi.org/10.1080/17460441.2020.1704729

14. Milletti F, Vulpetti A, F. M, A. V (2010) Predicting Polypharmacology by Binding Site Similarity:

https://doi.org/10.1021/ci1001263

576

15. Wood DJ, Vlieg J De, Wagener M, Ritschel T (2012) Pharmacophore fingerprint-based approach to binding site subpocket similarity and its application to bioisostere replacement. J Chem Inf Model 52:2031-2043. https://doi.org/10.1021/ci3000776

16. Kalliokoski T, Olsson TSG, Vulpetti A (2013) Subpocket analysis method for fragment-based drug discovery. J Chem Inf Model 53:131-141. https://doi.org/10.1021/ci300523r

17. Eguida M, Rognan D (2020) A Computer Vision Approach to Align and Compare Protein Cavities: Application to Fragment-Based Drug Design. J Med Chem 63:7127-7142. https://doi.org/10.1021/acs.jmedchem.0c00422

18. Zhou H, Cao H, Skolnick J (2021) FRAGSITE: A Fragment-Based Approach for Virtual Ligand Screening. J Chem Inf Model acs.jcim.0c01160. https://doi.org/10.1021/acs.jcim.0c01160

19. Rusu RB, Cousins S (2011) 3D is here: Point Cloud Library (PCL). In: 2011 IEEE International Conference on Robotics and Automation. IEEE, pp 1-4

20. Zhou Q-Y, Park J, Koltun V (2018) Open3D: A Modern Library for 3D Data Processing. arXiv:180109847. https://doi.org/10.1007/s00104-009-1793-x

21. Desaphy J, Azdimousa K, Kellenberger E, Rognan D (2012) Comparison and Druggability Prediction of Protein-Ligand Binding Sites from Pharmacophore-Annotated Cavity Shapes. J Chem Inf Model 
593 22. O’Connell J, Porter J, Kroeplien B, et al (2019) Small molecules that inhibit TNF signalling by

23. Kohlstaedt L, Wang J, Friedman J, et al (1992) Crystal structure at 3.5 A resolution of HIV-1 reverse

24. Brenner D, Blaser H, Mak TW (2015) Regulation of tumour necrosis factor signalling: live or let die.

600 Nat Rev Immunol 15:362-374. https://doi.org/10.1038/nri3834

25. Jochmans D (2008) Novel HIV-1 reverse transcriptase inhibitors. Virus Res 134:171-185. https://doi.org/10.1016/j.virusres.2008.01.003

603

26. Korb O, Stützle T, Exner TE (2009) Empirical Scoring Functions for Advanced Protein-Ligand 604

27. Ren J, Milton J, Weaver KL, et al (2000) Structural Basis for the Resilience of Efavirenz (DMP-266)

28. Wienken CJ, Baaske P, Rothbauer U, et al (2010) Protein-binding assays in biological liquids using 609

29. Jerabek-Willemsen M, André T, Wanner R, et al (2014) MicroScale Thermophoresis: Interaction analysis and beyond. J Mol Struct 1077:101-113. https://doi.org/10.1016/j.molstruc.2014.03.009 
Factor-Alpha is a dynamic process that is significantly altered by therapeutic antibodies. Sci Rep 10:9265. https://doi.org/10.1038/s41598-020-66123-5

31. Corti A, Fassina G, Marcucci F, et al (1992) Oligomeric tumour necrosis factor $\alpha$ slowly converts into inactive forms at bioactive levels. Biochem J 284:905-910.

https://doi.org/10.1042/bj2840905

32. Blevitt JM, Hack MD, Herman KL, et al (2017) Structural Basis of Small-Molecule Aggregate Induced Inhibition of a Protein-Protein Interaction. J Med Chem 60:3511-3517. https://doi.org/10.1021/acs.jmedchem.6b01836

33. Xiao H-Y, Li N, Duan JJW, et al (2020) Biologic-like In Vivo Efficacy with Small Molecule Inhibitors of TNF $\alpha$ Identified Using Scaffold Hopping and Structure-Based Drug Design Approaches. J Med Chem 63:15050-15071. https://doi.org/10.1021/acs.jmedchem.0c01732

34. Dietrich JD, Longenecker KL, Wilson NS, et al (2021) Development of Orally Efficacious Allosteric Inhibitors of TNF $\alpha$ via Fragment-Based Drug Design. J Med Chem 64:417-429. https://doi.org/10.1021/acs.jmedchem.0c01280

35. McMillan D, Martinez-Fleites C, Porter J, et al (2021) Structural insights into the disruption of TNFTNFR1 signalling by small molecules stabilising a distorted TNF. Nat Commun 12:582. https://doi.org/10.1038/s41467-020-20828-3

36. Weill N, Rognan D (2010) Alignment-free ultra-high-throughput comparison of druggable proteinligand binding sites. J Chem Inf Model 50:123-135. https://doi.org/10.1021/ci900349y

37. Schalon C, Surgand JS, Kellenberger E, Rognan D (2008) A simple and fuzzy method to align and compare druggable ligand-binding sites. Proteins Struct Funct Genet 71:1755-1778. https://doi.org/10.1002/prot.21858 
635

636

637

638

639

640

641

642

643

644

645

646

647

648

649

650

651

652

653

654

655

656

38. Lee HS, Im W (2016) G-LoSA: An efficient computational tool for local structure-centric biological studies and drug design. Protein Sci 25:865-876. https://doi.org/10.1002/pro.2890

39. Konc J, Janežič D (2010) ProBiS algorithm for detection of structurally similar protein binding sites by local structural alignment. Bioinformatics 26:1160-1168.

https://doi.org/10.1093/bioinformatics/btq100

40. Maggiora G, Vogt M, Stumpfe D, Bajorath J (2014) Molecular Similarity in Medicinal Chemistry. J Med Chem 57:3186-3204. https://doi.org/10.1021/jm401411z

41. Lo YC, Senese S, Damoiseaux R, Torres JZ (2016) 3D Chemical Similarity Networks for StructureBased Target Prediction and Scaffold Hopping. ACS Chem Biol 11:2244-2253. https://doi.org/10.1021/acschembio.6b00253

42. Rush TS, Grant JA, Mosyak L, Nicholls A (2005) A shape-based 3-D scaffold hopping method and its application to a bacterial protein-protein interaction. J Med Chem 48:1489-1495. https://doi.org/10.1021/jm040163o

43. Gaulton A, Bellis LJ, Bento AP, et al (2012) ChEMBL: a large-scale bioactivity database for drug discovery. Nucleic Acids Res 40:D1100-D1107. https://doi.org/10.1093/nar/gkr777

44. Burley SK, Bhikadiya C, Bi C, et al (2021) RCSB Protein Data Bank: powerful new tools for exploring 3D structures of biological macromolecules for basic and applied research and education in fundamental biology, biomedicine, biotechnology, bioengineering and energy sciences. Nucleic Acids Res 49:D437-D451. https://doi.org/10.1093/nar/gkaa1038

45. Bietz S, Urbaczek S, Schulz B, Rarey M (2014) Protoss: a holistic approach to predict tautomers and protonation states in protein-ligand complexes. J Cheminform 6:12. https://doi.org/10.1186/1758-2946-6-12 
657 46. Desaphy J, Bret G, Rognan D, Kellenberger E (2015) Sc-PDB: A 3D-database of ligandable binding 658 sites-10 years on. Nucleic Acids Res 43:D399-D404. https://doi.org/10.1093/nar/gku928

659 47. Desaphy J, Rognan D (2014) Sc-PDB-Frag: A database of protein-ligand interaction patterns for 660 bioisosteric replacements. J Chem Inf Model 54:1908-1918. https://doi.org/10.1021/ci500282c

661 48. Marcou G, Rognan D (2007) Optimizing fragment and scaffold docking by use of molecular 662 interaction fingerprints. J Chem Inf Model 47:195-207. https://doi.org/10.1021/ci600342e

663 49. Da Silva F, Desaphy J, Rognan D (2018) IChem: A Versatile Toolkit for Detecting, Comparing, and 664 Predicting Protein-Ligand Interactions. ChemMedChem 13:507-510.

665 https://doi.org/10.1002/cmdc.201700505

666 


\section{Supplementary Files}

This is a list of supplementary files associated with this preprint. Click to download.

- SI.pdf 